\title{
CLINICAL PRESENTATION OF PSORIASIS
}

\author{
F. AYALA \\ Division of Dermatology, Department of Systematic Pathology, University of Naples Federico II, Naples, Italy
}

\begin{abstract}
SUMMARY
Psoriasis is a chronic, inflammatory disease affecting 1-3\% of the world's population. Joints can be affected in up to $30 \%$ of patients. About one third of patients have either severe or moderate (involving more than $10 \%$ of body surface area) disease. Patients affected with extensive psoriasis have an impaired quality of life. Psoriasis has a large spectrum of clinical features and evolution, so no complete agreement on the classification of the clinical variants exists. Plaque psoriasis is the commonest form (more than $80 \%$ of affected patients). The course of plaque psoriasis varies. Spontaneous resolution is possible, but rarely occurs. Plaques tend to remain static or slowly enlarge. Flexural (inverse, intertriginous) psoriasis manifests with lesions thinner than those of plaque form with no or minimal scaling, and is localized in the skin folds. Guttate (eruptive) psoriasis has frequently a sudden onset and frequently appears abruptly after a bacterial or viral febrile episode of inflammation of the upper ways. Pustular and erythrodermic psoriasis are the most severe clinical variants. In the diffuse pustular form recurrent episodes of fever occur, followed by new outbreaks of pustules. Erythrodermic psoriasis corresponds to the generalized form of the disease. The entire skin is bright red and is covered by superficial scales. Fatigue, myalgia, shortness of breath, fever and chills may also occur. In sebopsoriasis (seborrheic dermatitis + psoriasis) the lesions tend to occur at the same sites as seborrheic dermatitis; greasy scales predominate, but silvery scales can be found in some areas. Nail psoriasis shows various features: nail pits; oil spots; subungual hyperkeratosis; onycholysis. Rare forms include psoriasis circinata, lip psoriasis and oral psoriasis. Differential diagnosis includes many other dermatological conditions.
\end{abstract}

Key words: Psoriasis, nail, quality of life

Psoriasis is a chronic, disabling, relapsing and remitting, inflammatory disease affecting $1-3 \%$ of the world's population. The onset can occur at any age; nevertheless, many patients are diagnosed, on average, during their 20s. Type I psoriasis, occurring before the age of 40 years, and type II, presenting later with a peak at 55-60 years, have been identified by some authors (1). The disorder affects males and females equally. Severity is variable, ranging from one or several cutaneous patches to severe exfoliation involving the entire skin (2). Psoriasis tends to be a symmetrical eruption. Joints can be affected in up to $30 \%$ of patients (3). As extension of the cutaneous lesions is concerned, about one third of patients have severe (involving more than $20 \%$ of body surface area [BSA] or moderate (involving 10-20\% of BSA) disease; remainders have a mild form, involving less than

Corresponding author:

Fabio Ayala, MD

Clinica Dermatologica

Università di Napoli Federico II

Via Pansini, 5 - 80131 Napoli, Italy

E-mail: ayala@unina.it
$10 \%$ of BSA. Moderate to severe psoriasis is nearly always associated with impaired quality of life. Psoriasis developing after injury of normal-appearing skin corresponds to the so-called Koebner phenomenon (or Koebner reaction): psoriatic, round or linear, lesions can occur a few days or weeks after different kinds of injury (excoriations, bites, burns, acupuncture, incisions, pressure, rubbing, linear scratches) as well as after different skin disorders (eczema, photosensitive disorders, herpes zoster, etc.).

Psoriasis has a large spectrum of clinical features and evolution $(4,5)$. No complete agreement on the classification of the clinical variants exists (6). Some authors believe that various patterns of presentation could represent separate diseases, as is the case of palmoplantar pustular psoriasis.

\section{PLAQUE PSORIASIS}

This is the commonest form of psoriasis that occurs in more than $80 \%$ of affected patients. In this form discoid, round or irregularly oval, well de- 


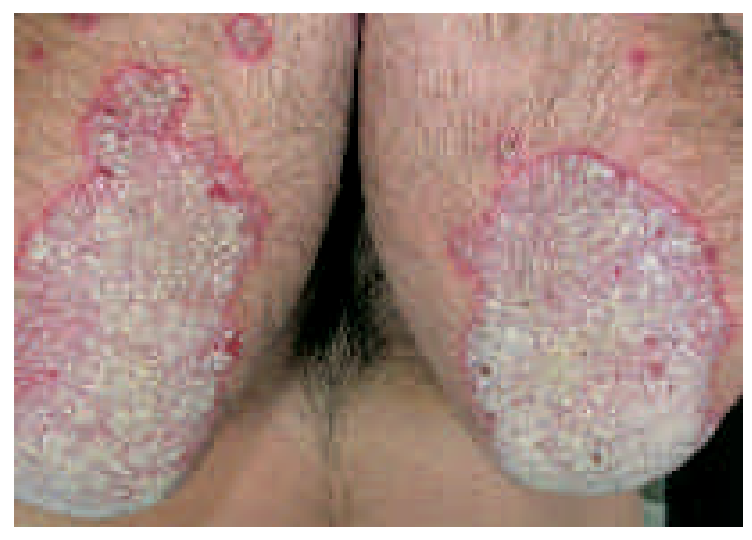

Figure 1 - Plaque psoriasis of the elbows; large lesions possibly derive from the confluence of small plaques, which are present on the forearms in this figure. Silvery scales and red edges characterize this chronic, stationary form.

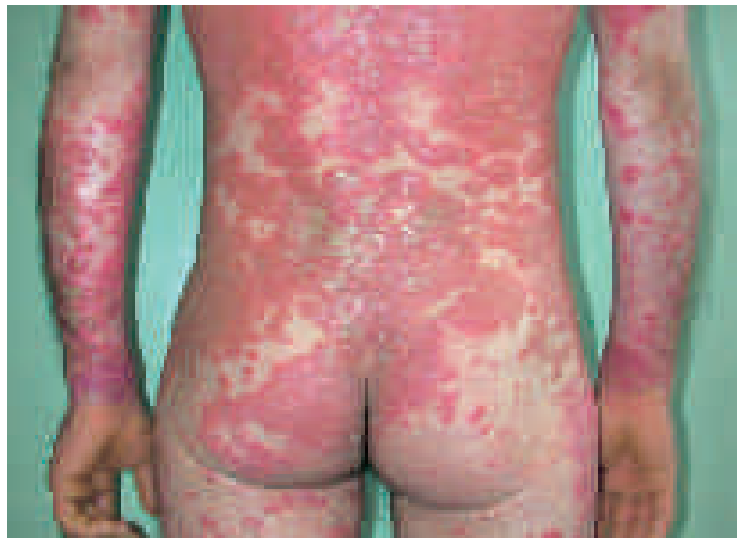

Figure 2 - Diffuse plaque psoriasis, showing confluent scaly lesions with typical morphology.

marcated raised plaques covered with silvery scales occur (Fig. 1). The underlying skin has a glossy, erythematous appearance. The plaque color may vary from red to white, according to the amount of scales. If untreated, a grayish white colour is sometimes observed. Lesions vary in size from $0.5 \mathrm{~cm}$ in diameter to large confluent areas (Fig. 2). They can appear anywhere on the cutaneous surface; scalp, extensor surfaces of elbows and knees, lumbar area are the most frequent sites. Plaques can derive from the confluence of small lesions (papules), sometimes resembling a land map, therefore the ancient term of "geographic psoriasis". Lesions tend to spare the face. An expanding, active rim accompanied by partial or total central clearing corresponds to the form called annular psoriasis, which tends to imply a better prognosis.
The course of plaque psoriasis varies. Spontaneous resolution is possible, but rarely occurs. Plaques tend to remain static or slowly enlarge. On clearing, a temporary hypopigmentation is frequent in the healed cutaneous areas.

Stable (stationary, inactive) psoriasis often shows a plaque-type pattern. Plaques then remain the same size even for months or years in patients undergoing no therapy. They are usually located in the above-mentioned areas of predilection.

\section{FLEXURAL (INVERSE, INTERTRIGINOUS) PSORIASIS}

Plaques are thin, unlike the typical thick plaque form, and this variety is localized in the skin folds (axillae, submammary regions, groins, natal cleft). Scaling is usually absent or negligible and the lesions show a glossy, sharply demarcated erythema. Maceration rarely occurs.

\section{SCALP PSORIASIS}

Plaques typically form on the scalp and along the hair margin. This is the commonest involved area and many patients discover they have psoriasis because of a dandruff-like desquamation of the scalp. There may be only one plaque or the disease can cover the entire scalp with thick, silvery scales on an erythematous base. Alopecia never ensues, even in the most diffuse forms. A particular variety, called pseudotinea amiantacea (Fig. 3) because of the resemblance of the scales to those seen in asbestos objects, is more frequent in children; scales are thicker than usually, moist at times, lacking the

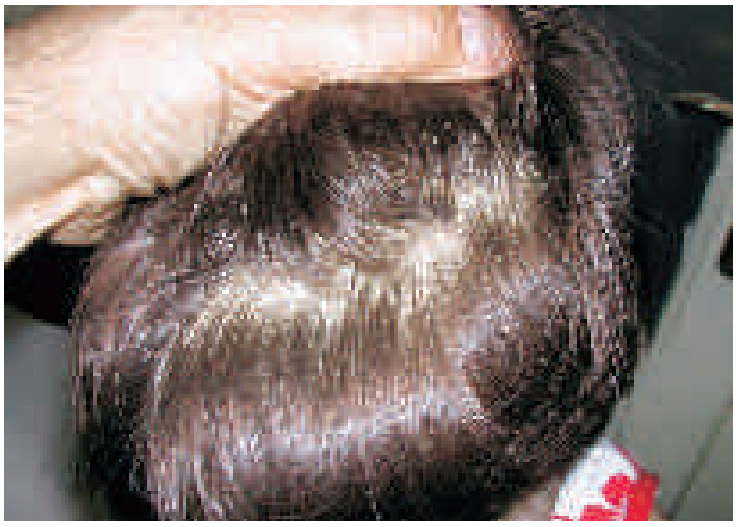

Figure 3 - Scalp psoriasis in a child, where the clinical aspect is that of pseudotinea amiantacea. 


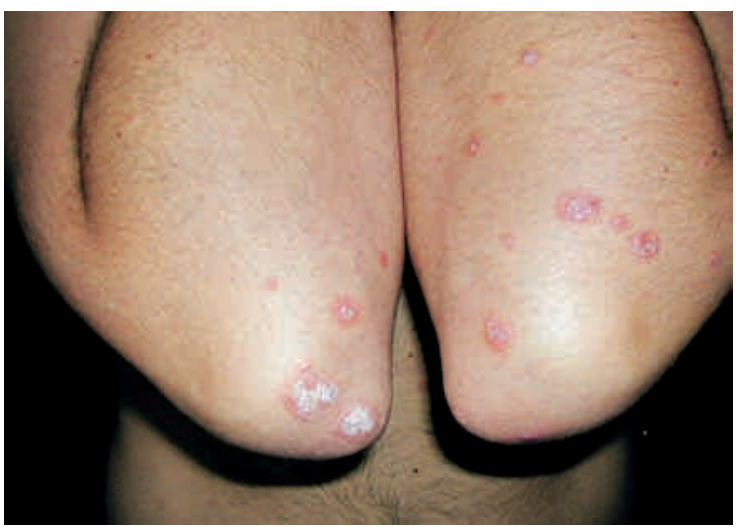

Figure 4 - Guttate psoriasis.

characteristic silvery aspect.

Scalp psoriasis doesn't assume a follicular distribution. Differently, psoriasis involving hair follicles of the trunk and limbs (follicular psoriasis) has a follicular pattern; children are more frequently affected.

\section{GUTTATE (ERUPTIVE) PSORIASIS}

Sudden onset is frequently reported by patients affected with this form of psoriasis. The small $(<1 \mathrm{~cm}$ in diameter) lesions usually occur over the upper trunk and proximal extremities (Fig. 4) and vary in number from a few scattered papules to hundreds. It typically affects children, adolescents and young adults, 2 to 4 weeks after a bacterial (or viral), febrile episode of inflammation of the upper ways. A preceding streptococcal infection (pharyngitis) is found in more than $50 \%$ of patients.

It must be distinguished from the common, small plaque form, corresponding to the relapse of the disorder after different therapies. If that is the case, the starting lesions often reappear in the same areas of the previously healed plaque lesions.

\section{PUSTULAR PSORIASIS}

Generalized pustular psoriasis (von Zumbush) is a rare, serious, acute variant of psoriasis. It may either be preceded by plaque psoriasis or arise $d e$ novo, but this form can be also triggered by local irritants (i.e. ultraviolet light from sunlight or phototherapy), withdrawal of systemic steroids, infections of the upper respiratory tract, drugs, etc. Many sterile, discrete, tiny pustules, 2- to 3-mm in diameter, evolve from an erythematous base, eventually coalescing in some areas into lakes of pus,

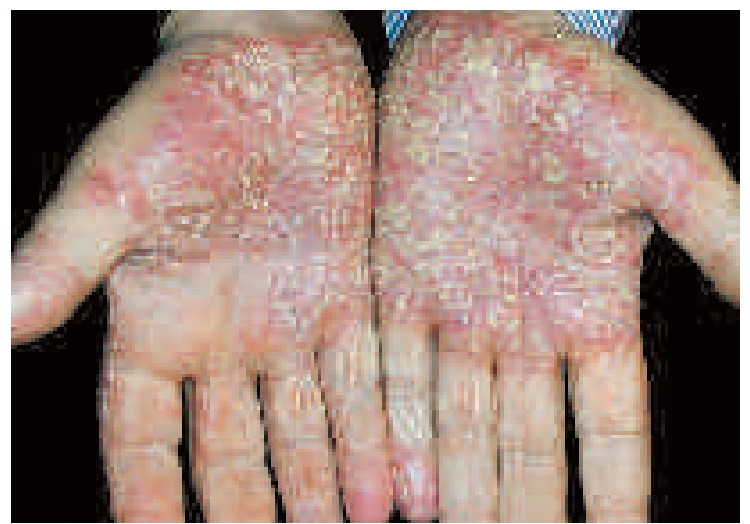

Figure 5 - Pustular psoriasis of the palms.

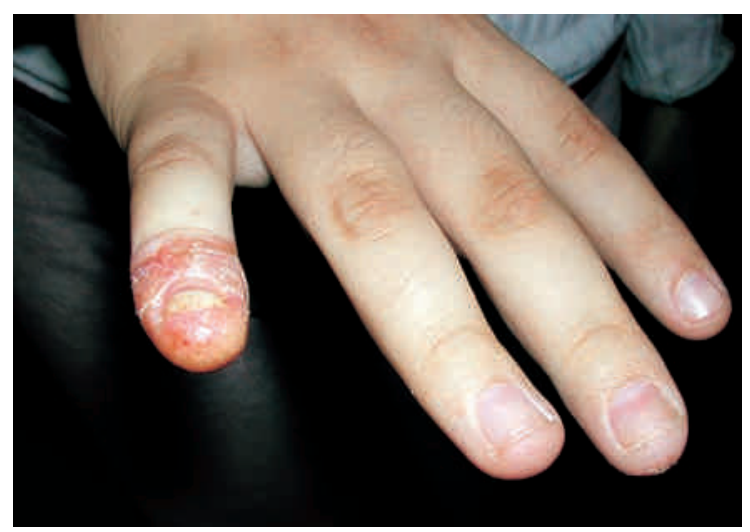

Figure 6 - Acrodermatitis continua of Hallopeau. Pustules on an erythematous base, nail dystrophy and recurrence in the same area are characteristic of this peculiar, acral type of psoriasis.

particularly in more severe cases. Pustules are easily ruptured. Usually recurrent episodes of fever occur, followed by new outbreaks of pustules. Weakness and weight loss are frequent systemic signs, often accompanied by leukocytosis and high erythrocyte sedimentation rate (ESR).

Localized pustular psoriasis (Barber) is the palmoplantar variant (Fig. 5).

Acrodermatitis continua (Hallopeau) is a rare form that usually affects fingers (Fig. 6) and toes around the nails and nail beds. Nail dystrophy and paronychial redness are often seen. Pustules tend to recur insistently in the same skin area. This form is particularly resistant to treatment.

\section{ERYTHRODERMIC PSORIASIS}

Erythroderma (i.e. erythema involving the entire skin) can be of psoriatic origin and corresponds to 


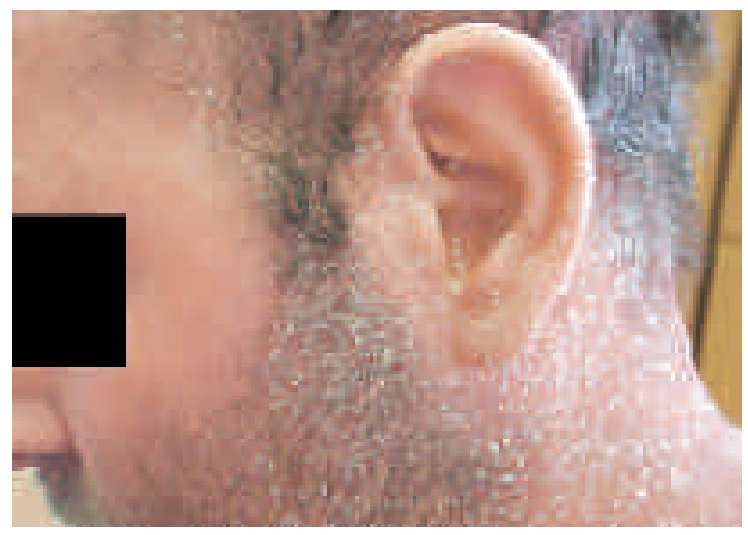

Figure 7 - Head and neck of a patient affected by psoriatic erythroderma. The entire skin was involved by intensely scaling erythema.

the generalized form of the disease, even if some authors believe that psoriasis involving more than $90 \%$ of the skin surface area can be referred to as erythroderma.

The skin is bright red and is covered by superficial scales (Fig. 7), which are quite different from the thick, adherent scales of classical, chronic plaque psoriasis. Erythema involving diffusely all the skin areas derives from generalized vasodilation, leading sometimes to excessive heat loss with consequent hypothermia.

Extravasation of proteins into the tissues due to vasodilation may cause edema of the limbs in patients with long-standing erythroderma. Fatigue, myalgia, shortness of breath, fever and chills may

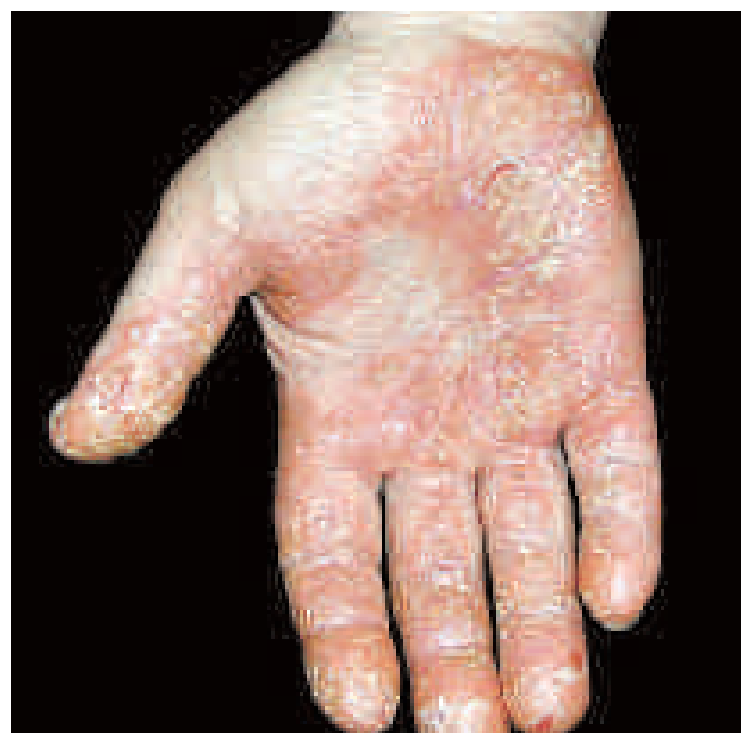

Figure 8 - Psoriasis of the palm, with marked hyperkeratosis and rhagades. also occur. Erythrodermic psoriasis tends to revert to extensive plaque disease. Generalized pustular psoriasis may revert to erythrodermic psoriasis when pustule formation stops. Abrupt withdrawals of systemic corticosteroids or methotrexate have been suggested, among others (e.g. sunburn), as possible trigger factors.

\section{ACRAL PSORIASIS}

The palms and soles may be the only sites involved in the manifestation of the disease, or they may be involved as part of a diffuse eruption or erythroderma. Hyperkeratosis (Fig. 8) is the main sign in some patients and is particularly evident at the margin of the heel areas, where rhagades can be seen. Redness is variable.

\section{SEBORRHEIC PSORIASIS}

In sebopsoriasis (seborrheic dermatitis + psoriasis) the lesions tend to occur at the same sites as seborrheic dermatitis: scalp, along the hair margin, eyebrows, nose, nasolabial folds (Fig. 9), pre-sternal and intertriginous areas. Because of its similarity in morphology, sometimes it may difficult to distinguish this peculiar form of psoriasis, especially when typical psoriatic plaques are not detectable in other skin areas.

Nevertheless, even if greasy scales predominate, silvery scales can be found in some areas, helping in making a correct clinical diagnosis.

This form corresponds to a typical Koebner phenomenon, seborrheic dermatitis leading to the occurrence of psoriasis in the same areas.

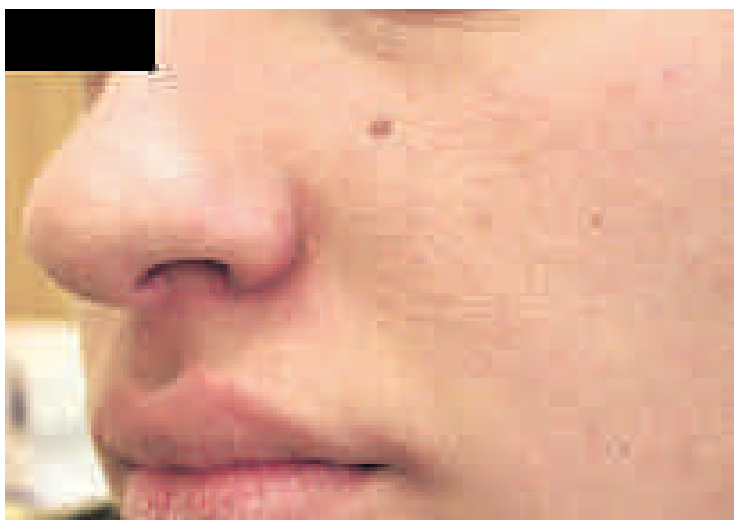

Figure 9 - Seborrheic psoriasis. In this patient a "greasy" appearance of the scales was evident over the forehead and the hair margin, too. 


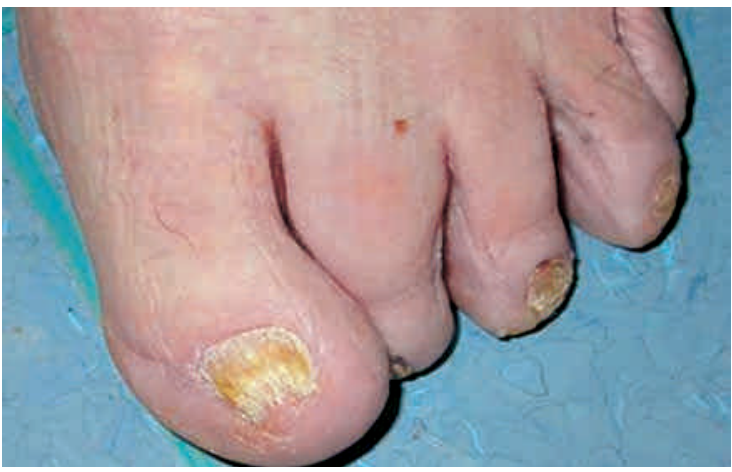

Figure 10 - Psoriasis of the toenails, manifesting as subungual hyperkeratosis. In these cases, mycology tests are needed to distinguish between psoriasis and fungal infections.

\section{NAIL PSORIASIS}

Nail changes are frequent (up to $50 \%$ of fingernails and $35 \%$ of toenails) in psoriasis (7). Nail involvement ranges from minor alterations in the nail plate to severe defects, up to loss of the nail plate in pustular forms of psoriasis. Proximal nail fold, nail matrix, nail bed and hyponychium can be involved, leading to different clinical aspects. Involved nails show various features depending on the location of the psoriatic process: nail pits (as a thimble); oil spots (yellowish or light brown, translucent discolorations under the nail plate); subungual hyperkeratosis (thickening of the nail plate with hyperkeratosis of the nail bed) (Fig. 10), onycholysis (separation of the distal nail plate from the nail bed); different, more or less severe onychodystrophy not easily classifiable. Rarely, nail psoriatic changes may occur without any skin lesion.

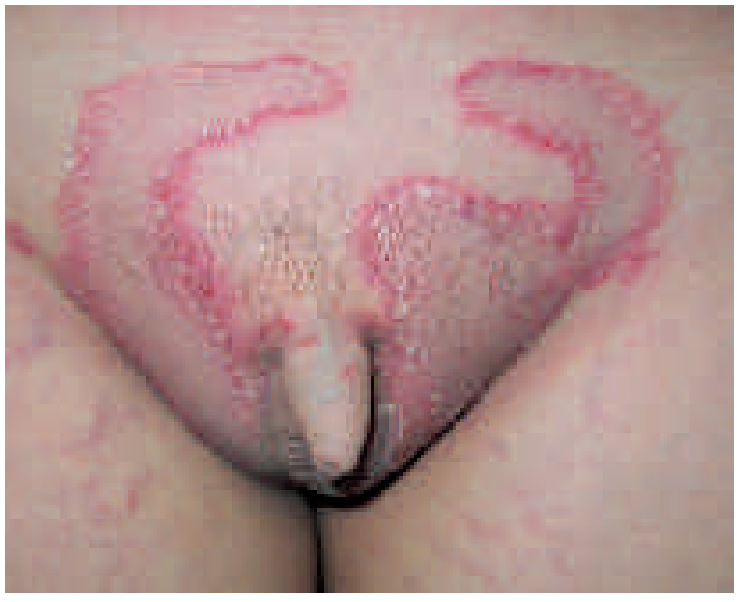

Figure 11 - Circinate psoriasis of the genital area in an infant.

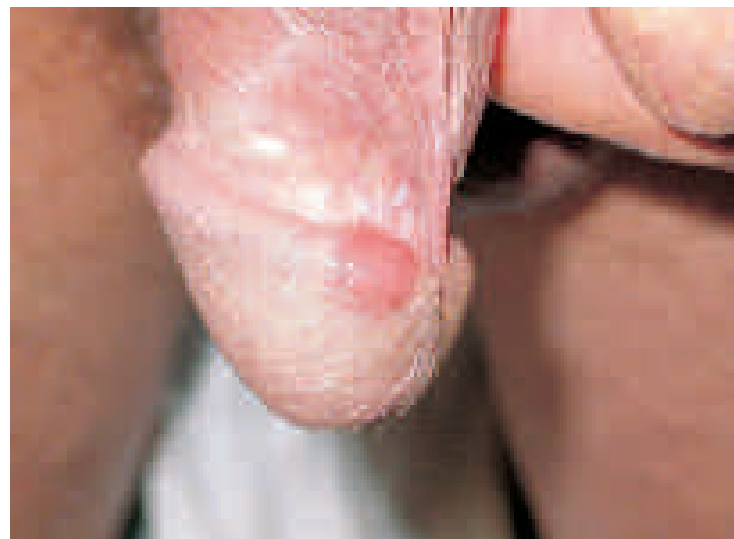

Figure 12 - Small plaque of psoriasis on the glans penis.

\section{RARE FORMS AND SOME SPECIFIC SITES}

Circinate psoriasis (Lapière) is a rare variant of the annular form, in which a figurate pattern is characteristic (arciform, serpiginous, "geographic") (Fig. 11).

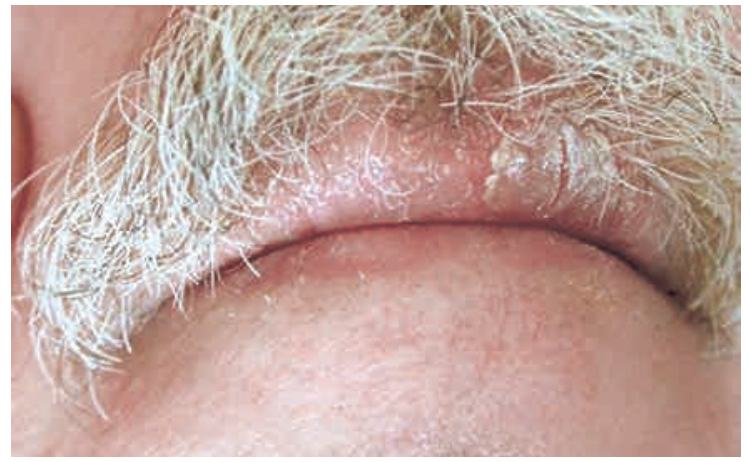

Figure 13 - Psoriasis of the lips.

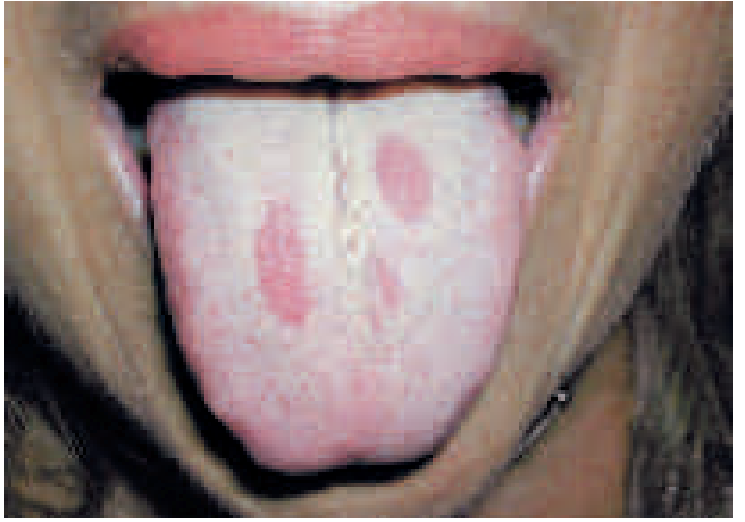

Figure 14 - Psoriasis in the form of denuded areas over the dorsal surface of the tongue. This patient had also plaque psoriasis involving the entire scalp, and acral psoriasis. 
If psoriasis only affects the glans, the commonest site of involvement is the proximal part (Fig. 12). The patches have a shiny appearance due to the ab-

\begin{tabular}{|c|c|}
\hline Psoriasis (type/skin area) & Main differential diagnoses \\
\hline Plaque & $\begin{array}{l}\text { Discoid (nummular) eczema } \\
\text { Lupus erythematosus, discoid } \\
\text { Mycosis fungoides (plaque type) } \\
\text { Pityriasis rubra pilaris }\end{array}$ \\
\hline Flexural & $\begin{array}{l}\text { Candidiasis } \\
\text { Contact dermatitis } \\
\text { Erythrasma } \\
\text { Seborrheic eczema } \\
\text { Tinea corporis }\end{array}$ \\
\hline Scalp & $\begin{array}{l}\text { Lupus erythematosus, discoid } \\
\text { Tinea capitis } \\
\text { Seborrheic eczema }\end{array}$ \\
\hline Guttate & $\begin{array}{l}\text { Pityriasis lichenoides } \\
\text { Pityriasis rosea } \\
\text { Seborrheic eczema } \\
\text { Syphilis, secondary } \\
\text { Tinea corporis }\end{array}$ \\
\hline Pustular & $\begin{array}{l}\text { Impetigo } \\
\text { Infected eczema (palmoplantar) } \\
\text { Migratory necrolytic erythema } \\
\text { Subcorneal pustular dermatosis }\end{array}$ \\
\hline Erythrodermic & $\begin{array}{l}\text { All other forms of erythroderma: } \\
\text { atopic eczema, cutaneous T cell } \\
\text { lymphoma (Sezary syndrome), } \\
\text { pityriasis rubra pilaris, actinic } \\
\text { reticuloid, etc }\end{array}$ \\
\hline Acral (palmoplantar) & $\begin{array}{l}\text { Chronic endogenous eczema } \\
\text { Contact dermatitis } \\
\text { Palmoplantar keratoderma } \\
\text { Palmoplantar pustulosis } \\
\text { Reiter's disease } \\
\text { Tinea manuum et pedis }\end{array}$ \\
\hline Nail & $\begin{array}{l}\text { Eczema (skin involvement } \\
\text { around the nails) } \\
\text { Lichen planus } \\
\text { Onycholysis (idiopathic, traumatic) } \\
\text { Onychomycosis }\end{array}$ \\
\hline
\end{tabular}

sence of scales, as is the case of flexural psoriasis. In circumcised males, some superficial scaling is sometimes present. It must be differentiated from erythroplasia of Queyrat, which is a form of epidermal carcinoma in situ.

If the lips are involved, silvery scales are often present on the vermilion (Fig. 13). Discoid lupus erythematosus and desquamative cheilitis must be differentiated.

Involvement of the oral cavity is still debated. Different clinical aspects have been reported (Fig.14), but geographic tongue seems to be the most frequent form, especially in patients with generalized pustular psoriasis.

The differential diagnosis depends on the form of psoriasis and the skin area involved (5).

\section{REFERENCES}

1. Henseler T, Christophers E. Psoriasis of early and late onset: characterization of two types of psoriasis vulgaris. J Am Acad Dermatol 1985; 13: 450-6.

2. Lebwohl M. Psoriasis. The Lancet 2003; 361: 1197204.

3. Scarpa R, Ayala F, Caporaso N et al. Psoriasis, psoriatic arthritis, or psoriatic disease? J Rheumatol 2006; 33: 210-2.

4. Fry L. Sites and clinical patterns. In: The encyclopedia of visual medicine series. An atlas of psoriasis. $2^{\text {nd }}$ edn. London and New York: Taylor \& Francis, 2004; 33-65.

5. Psoriasis. In: Pathology of the skin with clinical correlations (McKee PH, Calonje E, Granter SR eds). $3^{\text {nd }}$ edn. Philadelphia, USA: Elsevier Mosby, 2005; 195-206.

6. Griffiths CEM, Christophers E, Barker JNWN et al. A classification of psoriasis vulgaris according to phenotype. Br J Dermatol 2007; 156: 258-62.

7. Farber EM, Nall ML. The natural history of psoriasis in 5,600 patients. Dermatologica 1974; 148: 1-18. 